\title{
Trial of High Dosages of Dapsone in the Treatment of Tuberculoid Leprosy*
}

\author{
BIRENDRA N. PRASAD \\ Dermatologist and Venereologist, Rajendra Medical College and Hospital, \\ Ranchi, Bihar, India
}

\begin{abstract}
Two groups, each of 100 patients suffering from tuberculoid leprosy, were treated with different dosages of dapsone, the control group being given a conservative dosage of the drug and the study group a high dosage (1200 mg weekly). The patients in the high-dosage group showed more rapid regression of the disease, a lower incidence of relapse and early regression of the tingling, enlargement and tenderness of the nerve involved. Dapsone in a dosage of $1200 \mathrm{mg}$ weekly was well tolerated by these patients with active tuberculoid leprosy.
\end{abstract}

\section{Introduction}

Although the sulphones are toxic when taken in high doses, dapsone is the drug of choice in leprosy. The use of dapsone (DDS) may be followed by anaemia, which may be due to haemolysis, iron deficiency, or lack of some nutritional factor (Brownlee, 1948). Psychosis is another complication which may be encountered with high doses of the sulphones.

The low toxicity and therapeutic activity of dapsone as demonstrated by McEwen et al. (1941) encouraged Cochrane and fellow workers (1949) to use dapsone in the treatment of leprosy. The drug was thereafter given, but generally in low doses and some apprenension still persists regarding its use. However, Chang et al. (1952) reported a high safety level in the sulphones. Cochrane (1952) suggested that the basis of their proper use is to start with small doses of dapsone and gradually increase them to the maximum dose. The question that arises is: need dapsone be started in low dosage if the patients tolerate the drug well? If treatment is begun with the maximum therapeutic dose, it is conceivable that the disease would be arrested and cured more rapidly and complications averted, without allowing sequelae to develop.

The aim of the present study was to evaluate the response of patients with tuberculoid leprosy to a higher dosage schedule of dapsone than that conventionally used at present.

\section{Materials and Methods}

The 200 patients selected for the trial all had the tuberculoid type of leprosy and one or more infiltrated patches over the face, elbow, or leg, with enlargement

* Accepted for publication May, 1971. 
and tenderness of the regional nerve. They were of similar age-group and of both sexes and, for the purposes of the trial, patients with comparable disease and of similar age and sex were allotted to one of 2 groups of 100 each. In all cases the duration of the lesion was 6 months or more. Routine investigations of the urine and the blood (total white and red blood cell counts, differential count, and haemoglobin estimations) were done before starting treatment, and every 4 months thereafter. The lepromin test was positive in all patients, and examination of skin smears and nasal mucosa smears did not disclose any acid-fast organisms.

The first group of 100 patients was treated as follows: Dapsone, $50 \mathrm{mg}$, was given twice weekly, with $25 \mathrm{mg}$ increments every 2 weeks until $100 \mathrm{mg}$ twice a week was reached. The dose was then raised by $100 \mathrm{mg}$ every month until $300 \mathrm{mg}$ twice a week was reached. Then $100 \mathrm{mg}$ was given daily except Sunday. Tablets containing iron and a vitamin mixture were prescribed for all patients.

The patients in the second group were given the following treatment: Dapsone, $100 \mathrm{mg}$ twice daily, morning and night (except on Sundays). This dose was continued for 12 months. The same iron-vitamin tablets were given as in the first group. The patient was declared cured when the lesions had completely disappeared, together with nerve tenderness, or when repigmentation was almost complete.

After treatment for 12 months, the patients were examined every 4 months for 2 years, the routine investigations being repeated. The patients were instructed to report at once if they noticed any signs of relapse, or nerve tenderness, or the appearance of a new lesion.

\section{Results}

The patients on the higher dosages of dapsone showed marked improvement after taking the treatment for 6 weeks; in all patients, the infiltration in the lesions was the first abnormality to subside; then the erythema disappeared, and last of all pigmentation of the hypopigmented area returned. Nerve tenderness and enlargement diminished more slowly. In all the patients the lesions disappeared in 6 to 9 months. In regard to side-effects, only 9 patients complained of headache, loss of appetite, loss of taste, abdominal pain or anaemia. There was no relapse. In no patient did deformity develop. The urine remained normal throughout. Neuritis did not get worse during treatment.

In the group of patients receiving the conventional dosage schedule, the regression of the disease took longer, and it was only after 3 months of treatment that any improvement could be detected, with decrease in the erythema. The infiltration of the lesions took a year to disappear, and nerve tenderness even longer. Eight patients returned with relapse within 3 to 6 months of stopping treatment, 5 patients could not tolerate the drug, and 4 patients developed wasting of the small muscles of the hand.

In both groups only negligible changes occurred in the blood picture. The urine remained normal. No sensitivity rashes occurred, and no exacerbation of the leprosy.

\section{Discussion}

Though dapsone has been used in the treatment of leprosy since 1941, the best dose of the drug is still a matter of dispute. It is generally advocated that the 
treatment should begin with a low dose, which is gradually increased until the therapeutic level is attained. Recently the trend has been to give lower doses of dapsone. Leiker and Carling (1966) gave $200 \mathrm{mg}, 400 \mathrm{mg}$ and $800 \mathrm{mg}$ of dapsone weekly to 3 groups of patients respectively. None of the patients became bacteriologically negative. The frequency of lepromatous reactions remained unchanged in the patients given $800 \mathrm{mg}$ and $400 \mathrm{mg}$ weekly, while they increased in the group given $200 \mathrm{mg}$ weekly. Though admittedly based on a small number of cases, the study suggests that with small doses of dapsone, the results are in no way better than with larger doses, nor are the reactions in lepromatous leprosy lessened.

In the present study of patients with active tuberculoid leprosy, dapsone was given from the beginning in the high dose of $1200 \mathrm{mg}$ weekly. The drug was well tolerated, and the disease was rapidly controlled. Evidence of toxicity and intolerance to these high doses was found in only $9 \%$ of cases.

The advantages accruing from this high-dose regime are the following: (1) More rapid regression of the disease. (2) Rapid improvement in the subjective sensory sensations, such as tingling, and rapid reduction in observable enlargement and tenderness of the nerve involved. (3) Lower incidence of relapse. (4) Reduced incidence of toxicity and intolerance. (5) Less frequent occurrence of deformity.

\section{Acknowledgement}

I should like to thank Dr. N. K. Lal, Superintendent, Rajendra Medical College Hospital, Ranchi, who gave all the necessary help and facilities for this study.

\section{References}

Brownlee, G. (1948). Therapeutics and toxicology. Lancet ii, 131.

Chang, Y. T., Wolcott, R. R. and Doull, J. A. (1952). Sulphone therapy of leprosy. Med. Clin. N. Amer. 38, 599.

Cochrane, R. G. (1952). The chemotherapy of leprosy. Br. Med. J. ii, 1220.

Cochrane, R. G., Ramanujam, K., Paul, H. and Russell, D. (1949). Two and a half years experimental work on the sulphone group of drugs. Lepr. Rev. $20,4$.

Dharmendra and Chatterjee, K. R. (1955). Hydnosulphone in the treatment of leprosy. Lepr. in India 27, 230.

Leiker, D. L. and Carling, D. (1966). Low dosage of DDS. Lepr. Rev. 37, 27.

McEwen, A. D., Pizer, N. H. and Patterson, J. D. (1941). Preliminary trials on the administration of sulphonamides, E.O.S. Vet. Rec. 53, 429. 